\title{
Can Purified Omega-3 Polyunsaturated Fatty Acids Supplementation Act Blood Pressure Levels in Untreated Normal-High Blood Pressure Subjects with Hypertriglyceridemia?
}

\author{
Maria Leonarda De Rosa \\ Faculty of Medicine, University of Naples Federico II, Naples, Italy. \\ Email: mlderosa@unina.it \\ Received January $14^{\text {th }}, 2012$; revised February $18^{\text {th }}, 2012$; accepted March $9^{\text {th }}, 2012$
}

\begin{abstract}
Omega-3 ( $\omega-3)$ polyunsaturated fatty acids (PUFAs) from fish and fish oils appear to protect against coronary heart disease: their dietary intake is in fact inversely associated with cardiovascular disease morbidity/mortality in population studies. Recent evidence suggests that at least a part of this protective effect is mediated by a relatively small but significant decrease in blood pressure (BP) level. In fact, omega-3 PUFAs exhibit wide-ranging biological actions that include regulating both vasomotor tone and renal sodium excretion, partly competing with omega-6 PUFAs for common metabolic enzymes and thereby decreasing the production of vasoconstrictor rather than vasodilator and anti-inflammatory eicosanoids. PUFAs also reduce angiotensin-converting enzyme (ACE) activity, angiotensin II formation. We retrospectively evaluated the long-term effect of a omega-3 PUFAs supplementation on the blood pressure level of 91 hypertriglyceridemic subjects with untreated normal-high blood pressure that were prescribed a 3 grams omega-3 PUFAs supplementation in order to improve their plasma lipid pattern. After 24 months of treatment, systolic blood pressure (SBP) meanly decreased by $2.6+/-2.5 \mathrm{mmHg}(\mathrm{p}=0.001)$ and diastolic blood pressure (DBP) by $1.4+/-$ $3.1 \mathrm{mmHg}(\mathrm{p}<0.001)$, while basal heart rate decreased by $4.1+/-4.6 \mathrm{bpm}(\mathrm{p}<0.001)$. Both SBP and DBP reduction were significantly related to the baseline SBP $(p<0.001)$ and DBP $(p<0.001)$, respectively. Diastolic blood pressure change was also inversely related to the patient's age $(p=0.004)$. No significant difference was perceived in the metabolic syndrome subgroup. In our retrospective study, highly purified omega-3 PUFAs long-term supplementation is associated with a significant reduction in SBP, DBP, and basal heart rate in hypertriglyceridemic patients with normal-high blood pressure. The main determinants of the omega-3 PUFAs anti-hypertensive effect appear to be the basal blood pressure level and age. Future research will clarify if omega-3 PUFAs supplementation could improve the antihypertensive action of specific blood pressure lowering drug classes and of statins.
\end{abstract}

Keywords: Omega-3PUFAs; Blood Pressure; Hypertriglyceridemia

\section{Introduction}

Dietary factors play a major role in the pathogenesis of essential hypertension. In fact several lines of evidence suggest that supplementation of diet with omega-3 polyunsaturated fatty acids ( $\omega$-3 PUFAs), commonly referred to as fish oil, may reduce blood pressure; hence, it is likely that animals fed $10 \%$ safflower oil with $30 \%$ casein had higher amounts of free radicals and pro inflammatory cytokines and developed hypertension as a consequence of low-grade systemic inflammation [1,2]. These evidences are relevant to patients with chronic renal failure; high-protein diet enhances oxidative stress and interferes with the production of lipoxins, resolvins, pro- tectins, and maresins (anti-inflammatory compounds) that could adversely affect the underlying renal disease [3]. The dietary intake of $\omega$-3 PUFAs is in fact inversely associated with cardiovascular disease morbidity/mortality in population studies [4]. However, most clinical trials of $\omega$-3 PUFAs supplementation have been of insufficient size to detect relevant blood pressure changes $[5,6]$. In the body there are two types of essential fatty acid (EFA), the $\omega-6$ linoleic acid and $\omega-3 \alpha$-linolenic acid, metabolized by the same set of enzyme to give raise to arachidonic acid and eicosapentaenoic acid (EPA), the precursors of eicosanoids. Arachidonic acid, EPA and docosahexaenoic acid (DHA) (formed from eicosapentenoic acid) also form precursors to the anti-inflamma- 
tory compounds that participate in several physiological and pathological processes [2].

The present study was designed to investigate the relation between dietary supplements of purified $\omega$-3 PUFAs (EPA + DHA with ratio 0.9/1.5, respectively) 3 grams /day and blood pressure in untreated normal-high blood pressure population.

\section{Methods}

From our cardiovascular prevention ambulatory, nonsmoking subjects with untreated normal-high essential hypertension, but no other clinically evident disease, except that the hypertriglyceridemia, were screened for inclusion in the our retrospectively study. In order to evaluate the long-term effect of an $\omega$-3 PUFAs supplementation on the blood pressure level, for two years we studied ninety one patients, who gave signed consent. They were prescribed a 3 grams/day $\omega-3$ PUFAs supplementation to improve their level of plasma lipids. Table 1 shows general characteristics of study population at baseline; while Table 2 shows the study flowchart.

\subsection{Clinic Blood Pressure and Pulse Rate Measurement}

Participants sat quiet for at least $5 \mathrm{~min}$ and arm circumference was measured to determine the appropriate cuff size. Two blood pressure readings were auscultated with a mercury manometer at 2 min intervals by a certified staff, between which pulse rate was palpated for $30 \mathrm{sec}$. Blood pressure reading were averaged across the two appointments and are referred to hereafter as "clinic" systolic and diastolic blood pressure.

\subsection{4-Hambulatory Blood Pressure and Pulse Rate Measurement}

Participants wore an ambulatory blood pressure device for $24 \mathrm{~h}$. The device was programmed to automatically record blood pressure every 30 min between 7 a.m. and 11 p.m. and every hour between 11 p.m. and 7 a.m. Subjects noted the time they went to bed at night and the time of arising in the morning. Using this information, device data were averaged during "awake" and "asleep" time periods.

During the supplementation period the subjects were asked to reduce the intake of fat in their usual foods to avoid changes in weight. If they used statins only a stabilized dosage for at least 5 months and without changing other drug. Participants met with the researchers six times over two years. In each session, they got their blood pressure checked, provided a blood sample, and reported everything they had eaten and drunk in the past 24 hours. We also considered other factors, including participants'
Table 1. General characteristics of study population at the baseline (values expressed as mean $\pm \mathrm{SD}$ ).

\begin{tabular}{cc}
\hline Age (years) & $58 \pm 12$ \\
\hline Sex male & $72 \%$ \\
female & $28 \%$ \\
Body mass index $\left(\mathrm{Kg} / \mathrm{m}^{2}\right)$ & $26.9 \pm 3.91$ \\
W C (cm) & $91.09 \pm 10.09$ \\
SBP (mmHg) & $138.7 \pm 5.0$ \\
DBP $(\mathrm{mmHg})$ & $90.3 \pm 1.6$ \\
HR (b/m) & $72.0 \pm 2.0$ \\
TC (mg/dL) & $232.75 \pm 30.92$ \\
HDL-C (mg/dL) & $54.46 \pm 17.48$ \\
LDL-C (mg/dL) & $128.55 \pm 26.93$ \\
TG (mg/dL) & $248.72 \pm 48.5$ \\
FPG (mg/dL) & $104.90 \pm 18.14$ \\
HbAlc (mg/dL) & $5.8 \pm 0.6$ \\
Creatinine (mg/dL) & $0.98 \pm 1.8$ \\
\hline
\end{tabular}

Abbreviations: WC: waist circumference; SBP: systolic blood pressure; DBP: diastolic blood pressure; HR: heart rate; TC: total cholesterol; TG: triglycerides; HDL-C: high density lipoprotein cholesterol; LDL-C: low density lipoprotein cholesterol; FPG: fasting plasma glucose.

Table 2. Study flowchart.

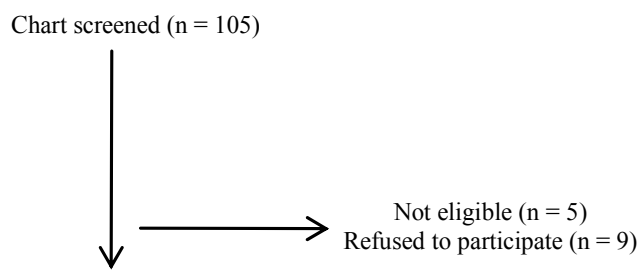

Studied $(\mathrm{n}=91)$

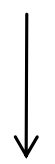

Adverse event $(\mathrm{n}=1)$

Patient withdrawal $(\mathrm{n}=1)$

Non compliance $(\mathrm{n}=1)$

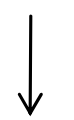

Completed study $(\mathrm{n}=88)$

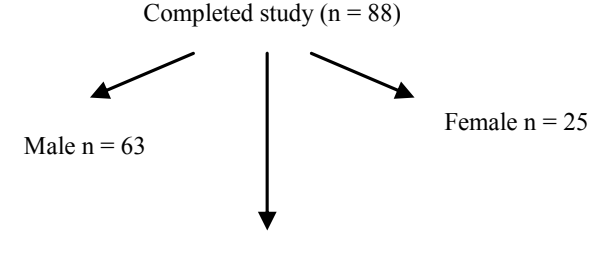

Patients with metabolic syndrome $n=33$ 
age, gender, alcohol consumption, physical activity, dietary restrictions, supplements, and medications.

\section{Statistics}

No parametric distribution for any of the outcome variables was assumed because of small sample size. Wilcoxon's exact rank sum test and Fisher's exact test (for continuous and categorical outcomes) compared the baseline characteristics as well as other outcomes. Only subjects who completed the study and had both baseline and final measurements were included in the analysis. A p-value of $<5 \%$ was considered statistically significant. We did not adjust p-values for multiple comparisons since all the analyses were exploratory in nature. Because this was a retrospectively study no power calculations were performed, and all findings need to be confirmed in a larger clinical trial. All statistical analyses were performed using SAS software (Italy) [7,8].

\section{Results}

Eighty eight subjects tolerated the supplements, as it showed in Table 2, the flowchart of study. One subject dropped out of follow-up for reasons unrelated to the study, other one for no compliance to the supplementation 3 grams/day intake of omega-3 fatty acids. One patient had dyspepsia as adverse event. None of the patients had an increased susceptibility to bruising. Body weight were similar among the study and did not change significantly during the course of the study. Table 3 shows the changes of study population's parameters during the study. The subjects completing the study had no changes in values for routine blood counts or chemistries, except for a reduction in fasting triglyceride levels. Plasma lipid values significantly changed from the baseline after twenty four months of treatment; total cholesterol changed from $232.75 \pm 30.92 \mathrm{mg} / \mathrm{dl}$ to $209.1 \pm 14 \mathrm{mg} / \mathrm{dl}(\mathrm{p}<0.001)$,
HDL-cholesterol from $54.46 \pm 17.48 \mathrm{mg} / \mathrm{dl}$ to $64.9 \pm 15$ $\mathrm{mg} / \mathrm{dl}(\mathrm{p}<0.001)$, LDL-cholesterol from $128.55 \pm 26.93$ $\mathrm{mg} / \mathrm{dl}$ to $116.98 \pm 0.2 \mathrm{mg} / \mathrm{dl}(\mathrm{p}<0.001)$ and triglycerides from $248.72 \pm 48.5 \mathrm{mg} / \mathrm{dl}$ to $136.1 \pm 0.9 \mathrm{mg} / \mathrm{dl}(\mathrm{p}<$ $0.0001)$. We did not observe any specific differences that could lead to the creation of subgroup affected or not affected by metabolic syndrome. After twenty months of omega-3 PUFAs treatment, the basal heart rate also significantly decreased from $72.0 \pm 2.0 \mathrm{bpm}$ to $66.1 \pm 0.9$ $\mathrm{bpm}(\mathrm{p}<0.0001)$. Participants with 3 grams/day intake of omega-3 fatty acids tended to have the lowest blood pressure with significantly greater reduction: SBP meanly decreased by $-6.5 \pm 2.2 \mathrm{mmHg}(\mathrm{p}<0.04)$, DBP by -4.4 $\pm 0.9 \mathrm{mmHg}(\mathrm{p}<0.04)$. Systolic pressure decreased earlier than diastolic pressure during supplementation with 3 grams/day intake of omega-3 fatty acids. Diastolic blood pressure change was also inversely related to the patient's age $(p=0.004)$.The effect of fish oil on blood pressure seemed to have reached a plateau by twenty months (Figure 1). That pattern was particularly strong in this population who didn't have high blood pressure and who wasn't already on restricted diets or medications to control its blood pressure. We note that omega-3 fatty acids didn't appear to drastically slash blood pressure. But every small reduction in blood pressure counts, including foods rich in omega-3 fatty acids as part of a healthy diet, may have blood pressure benefits. Figure 2 shows the graphical representation of the response to the supplementation dose of omega-3 PUFAs and the time needed for acting on clinical effects. Both SBP and DBP reduction are significantly related to the baseline SBP and DBP, respectively, independently from the patient $\operatorname{sex}(p<0.04)$. Baseline triglycerides are significantly associated with BP change, independently from the patient sex $(\mathrm{p}<0.0001)$. Heart rate variation was significantly related to both BP and triglycerides parameters, $(\mathrm{p}<$ 0.0001 and $\mathrm{p}<0.001$, respectively).

Table 3. Changes of study population's parameters during the study (values expressed as mean \pm SD).

\begin{tabular}{cccccc}
\hline & Baseline & 6 & Months & 12 & Months \\
\hline Body mass index (Kg/m²) & $26.9 \pm 3.91$ & $25.8 \pm 4.4$ & $25.9 \pm 4.3$ & $25.9 \pm 4.5$ & $25.4 \pm 4.4$ \\
(WC (cm) & $91.09 \pm 10.09$ & $92.02 \pm 7.9$ & $90.4 \pm 9.0$ & $89.9 \pm 9.1$ & $89.9 \pm 9.0$ \\
HR (b/m) & $72.0 \pm 2.0$ & $69.9 \pm 1.0^{*}$ & $68.9 \pm 0.8^{*}$ & $66.9 \pm 0.7^{\infty}$ & $66.1 \pm 0.9^{\infty}$ \\
TC (mg/dL) & $232.75 \pm 30.92$ & $223.7 \pm 27.4^{*}$ & $218.6 \pm 26.4^{*}$ & $215.30 \pm 20.0^{*}$ & $209.1 \pm 14^{*}$ \\
HDL-C (mg/dL) & $54.46 \pm 17.48$ & $62.5 \pm 12.0^{*}$ & $60.9 \pm 12.0^{*}$ & $63.4 \pm 13.0^{*}$ & $64.9 \pm 15^{*}$ \\
LDL-C (mg/dL) & $128.55 \pm 26.93$ & $122.34 \pm 8.6^{*}$ & $122.9 \pm 8.8^{*}$ & $117.57 \pm 5.0^{*}$ & $116.98 \pm 0.2^{*}$ \\
TG (mg/dL) & $248.72 \pm 48.5$ & $194.3 \pm 34.6^{*}$ & $179.0 \pm 28.0^{*}$ & $152.30 \pm 10.0^{*}$ & $136.1 \pm 0.9^{\infty}$ \\
FPG (mg/dL) & $104.90 \pm 18.14$ & $102.8 \pm 16.9$ & $101.87 \pm 13.2$ & $101.2 \pm 12.0$ & $101.0 \pm 10.8$ \\
HbA1c (mg/dL) & $5.8 \pm 0.6$ & $5.7 \pm 0.7$ & $5.6 \pm 0.7$ & $5.5 \pm 0.6^{*}$ & $5.5 \pm 0.5^{*}$ \\
Creatinine (mg/dL) & $0.98 \pm 1.8$ & $0.98 \pm 1.9$ & $0.98 \pm 1.9$ & $0.98 \pm 1.2$ & $0.98 \pm 1.3$ \\
\hline
\end{tabular}

P value: ${ }^{*} \mathrm{p}<.001 ;{ }^{\infty} \mathrm{p}<.0001$; Abbreviations: WC: waist circumference; HR: heart rate; TC: total cholesterol; TG: triglycerides; HDL-C: high density lipoprotein cholesterol; LDL-C: low density lipoprotein cholesterol; FPG: fasting plasma glucose. 


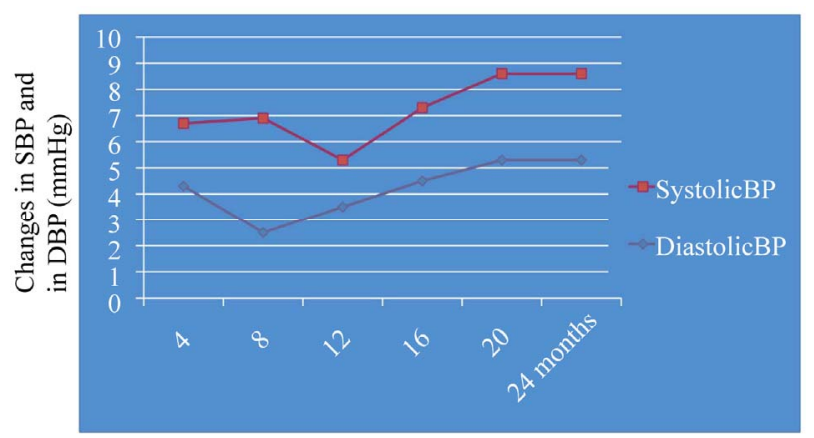

Figure 1. Graphical representation of BP changes during the fish oil supplementation. Systolic blood pressure change: $-6.5 \pm 2.2$; Diastolic blood pressure change: $-4.4 \pm 0.9$; $\mathrm{P}<$ 0.04 .



Figure 2. Graphical representation of the response to the supplementation dose of omega-3 PUFAs and the time needed for acting on clinical effects.

\section{Discussion}

According to the American Heart Association, Omega-3 fatty acids benefit the heart of healthy people, and those at high risk of - or who have-cardiovascular disease. Research has shown that omega-3 fatty acids decrease risk of arrhythmias (abnormal heartbeats), which can lead to sudden death. Omega-3 fatty acids also decrease triglyceride levels, slow growth rate of atherosclerotic plaque, and produce modest reductions in blood pressure (slightly). The omega-3 fatty acids in fish oil seem to be able to expand blood vessels, and this brings blood pressure down [9]. It appears that omega-3 PUFAs confer cardiovascular benefits largely through DHA and EPA enrichment of membrane phospholipids [10]. Ventura et al. showed that in patient after cardiac transplantation with Cycosporine-induced hypertension omega-3 fatty acids produced vasodilatation, reducing blood pressure [11].

In according to Theis F. et al. association of omega-3 polyunsaturated fatty acids stabilized the atherosclerotic plaques improving arterial and endothelial function [12], and in according to Din et al. they reduced platelet aggregation [13]. Our results are in according to Mozaffarian et al. who showed that the antiplatelet, antiinflammatory, and triglyceride-lowering effects of omega-3 PUFAs require relatively higher doses of DHA and EPA (e.g., 3 to $4 \mathrm{~g} /$ day), whereas some of the antiarrhythmic effects, improvement in heart failure can be achieved at lower doses (500 to $1000 \mathrm{mg}$ /day) [14].

Although the effects of omega-3 PUFAs on C-reactive protein levels have been inconsistent [15], these agents have been shown to suppress production of pro-inflammatory cytokines such as interleukin-1B, interleukin-6, and tumor necrosis factor-alpha [16]. When administered to obese patients, $1.8 \mathrm{~g}$ of EPA increased the levels of adiponectin, which can reduce inflammation and improve insulin sensitivity [17], in addition to the potential beneficial heart failure effects discussed earlier. Although benefits on the autonomic nervous system are well established and are reviewed earlier, studies in patients undergoing heart transplantation suggest that omega-3 PUFAs can reduce heart rate independently of vagal activation, in addition to reducing mean arterial pressure and systemic vascular resistance by $25 \%$ and reducing left ventricular hypertrophy and improving diastolic function [18].

The optimal doses and ratios of DHA to EPA are difficult to decipher. DHA is far more abundant than EPA in the myocardium [13]. DHA alone or in combination with EPA may be more important for protection against dysrhythmias and cardiovascular disease than EPA alone. Although the beneficial effects on dysrhythmias seem to occur at lower doses, the relative risk of cardiovascular disease has been shown to be related with baseline blood levels of omega-3 PUFAs [19-21]. In addition, other surrogate CV markers (arterial pressure, endothelial relaxation and attenuated vascular relaxation, and lipoproteins) may be more improved with high doses of DHA than with similar doses of EPA [22]. Our results showed that omega-3 fatty acids appear to have a small, dose dependent, hypotensive effect, the extent of which seems to be dependent on the degree of hypertension. In a metaanalysis, Morris et al. found a significant reduction in blood pressure of 3.4/2.0 $\mathrm{mmHg}$ in studies with hypertensive subjects who consumed $5.6 \mathrm{~g} /$ day of omega-3 fatty acids [23]. However this may happen at the cost of adverse reactions in such a high dose. Omega-3 fatty acids in such high doses are not recommended for treatment of hypertension [24]. In our study in according to previously an increased intake of Omega-3 fatty acids since to 3 gram/day reduced blood pressure by $5 \%$ to $10 \%$. In our previous studies we demonstrated that when sustained, weight loss, albeit modest, led to a $22 \%$ reduc- 
tion in the risk for hypertension among middle-aged adults, and a $26 \%$ reduction in risk in older adults without difference of sex $[25,26]$. DHA may have greater benefits than EPA. However, high intakes of omega-3 fatty acids per day may be necessary to obtain clinically relevant effects, and at this dose level, there is an increased risk of bleeding [27]. Therefore, a qualified healthcare provider should be consulted prior to starting treatment with supplements. In according to Mori et al. clinical trials in humans have shown that eicosapentaenoic acid (EPA) and docosahexaenoic acid (DHA) have different haemodynamic properties; docosahexaenoic acid may be more favourable in lowering blood pressure and heart rate, as well as improving vascular function [28]. However, the effects of EPA and DHA may also differ depending on the target population [28].

\section{Conclusion}

Our analyses indicate that the diet supplementation with a relatively high dose of $\omega-3$ PUFAs $3 \mathrm{~g} /$ die, can lead to clinically relevant blood pressure reduction in individuals with untreated normal-high hypertension with and without metabolic syndrome, without difference of sex and age, during 2 years of retrospectively study. In this study there was no significant change in weight, and waist circumference nor in fasting plasma glucose. Furthermore we believe that the field of genomics may be important in explaining dietary response via "Mendelian randomization" and interactions between diet and genotype. Research needs to be conducted in different part of the world to better understand the responses of different population to diet. Further studies will be needed to address the potential benefits of assessing blood levels of omega3 PUFAs to determine its role in dosing adjustments and its value in cardiovascular protection. Convincing evidence from extensive research over the past three decades points out the potential beneficial effects of omega3 PUFAs in primary prevention.

\section{REFERENCES}

[1] D. P. Begg, A. J. Sinclair, L. A. Stahl, M. L. Garg, M. Jois and R. S. Weisinger, "Dietary Protein Level Interacts with $\omega-3$ Polyunsaterated Fatty Acid Deficiency to Induce Hypertension," American Journal of Hypertension, Vol. 23, No. 2, 2010, pp. 125-128.

[2] U. N. Das, "Essential Fatty Acids and Their Metabolites Could Function as Endogenous HMG-CoA Reductase and ACE Enzyme Inhibitors, Anti-Arrhythmic, Anti-Hypertensive, Anti-Atherosclerotic, Anti-Inflammatory, Cytoprotective and Cardioprotective Molecules," Lipids in Health and Disease, Vol. 7, 2008, p. 37.

[3] U. N. Das, "Interaction of Polyunsaturated Fatty Acids with Dietary Protein and Its Relationship to the Patho- genesis of Hypertension," American Journal of Hypertension, Vol. 23, No. 2, 2010, pp. 111-112.

[4] D. Mozaffarian, R. Micha and S. Wallace, "Effects on Coronary Heart Disease of Increasing Polyunsaturated Fat in Place of Saturated Fat: A Systematic Review and Meta-Analysis of Randomized Controlled Trials," PLoS Medicine, Vol. 7, 2010, Article ID: 1000252.

[5] J. M. Geleijnse, E. J. Giltay, D. E. Grobbee, A. R. Donders and F. Kok, "Blood Pressure Response to Fish Oil Supplementation: Metaregression Analysis of Randomized Trials," Journal of Hypertension, Vol. 20, 2002, pp. 1493-1499. doi:10.1097/00004872-200208000-00010

[6] C. Borghi and A. F. G. Cicero, "Blood Pressure Modulating Properties of Omega-3 Polyunsaturated Fatty Acids(PUFA). Another PUFA Heart Protective Effect?" High Blood Pressure and Cardiovascular Prevention, Vol. 14, No. 2, 2007, pp. 1-7. doi:10.2165/00151642-200714020-00001

[7] D. G. Altman, "Practical Statistics for Medical Research," Chapman and Hall, London, 1991.

[8] C. R. Mehta, N. R. Patel and A. A. Tsiatis, "Exact Significance Testing to Establish Treatment Equivalence with Ordered Categorical Data," Biometrics, Vol. 40, No. 3, 1984, pp. 819-825. doi:10.2307/2530927

[9] A. H. Lichtenstein, L. J. Appel, M. Brands, et al., "Diet and Lifestyle Recommendations: Revision 2006. A Scientific Statement from the American Heart Association Nutrition Committee," Circulation, Vol. 114, No. 1, 2006, pp. 82-96.

[10] W. S. Harris, "Omega-3 Fatty Acids and Cardiovascular Disease a Case for Omega-3 Index as a New Risk Factor," Pharmacological Research, Vol. 55, No. 3, 2007, pp. 217-223.

[11] H. O. Ventura, R. V. Milani, C. J. Lavie, et al., "Cycosporine-Induced Hypertension: Efficacy of Omega-3 Fatty Acids in Patients after Cardiac Transplantation," Circulation, Vol. 88, 1993, pp. 11281-11285.

[12] F. Theis, J. M. Garry, P. Yaquoob, et al., "Association of n-3 Polyunsaturated Fatty Acids with Stability of Atherosclerotic Plaques: A Randomized Controlled Trial," Lancet, Vol. 3161, 2003, pp. 477-485. doi:10.1016/S0140-6736(03)12468-3

[13] J. N. Din, S. A. Harding, C. J. Valerio, et al., "Dietary Intervention with Oil Rich Fish Reduces Platelet-Monocyte Aggregation in Man," Atherosclerosis, Vol. 197, No. 1, 2008, pp. 290-296.

[14] D. Mozaffarian and E. B. Rimm, "Fish Intake, Contaminants, and Human Health: Evaluating the Risk and the Benefits," JAMA, Vol. 296, No. 15, 2006, pp. 1885-1899. doi:10.1001/jama.296.15.1885

[15] T. Madsen, E. B. Schmidt and J. H. Christensen, "The Effect of n-3 Fatty Acids on C-Reactive Protein Levels in Patients with Chronic Renal Failure," Journal of Renal Nutrition, Vol. 17, No. 4, 2007, pp. 258-263.

[16] G. Zhao, T. D. Etherton, K. R. Martin, P. J. Gilles, S. G. West and P. M. Kris-Etherton, "Dietary Alpha-Linolenic Acid Inhibits Proinflammatory Cytokine Production by 
Peripheral Blood Mononuclear Cells in Hypercholesterolemic Subjects," American Journal of Clinical Nutrition, Vol. 85, No. 2, 2007, pp. 385-391.

[17] M. Itoh, T. Suganami, N. Satoh, et al., "Increased Adiponectin Secretion by Highly Purified Eicosapentaenoic Acid in Rodent Models of Obesity and Human Obese Subjects," Arteriosclerosis, Thrombosis, and Vascular Biology, Vol. 27, No. 9, 2007, pp. 1918-1925. doi:10.1161/ATVBAHA.106.136853

[18] W. S. Harris, M. Gonzales, N. Laney, A. Sastre and A. M. Borkon, "Effects of Omega-3 Fatty Acids on Heart Rate in Cardiac Transplant Recipients," American Journal of Cardiology, Vol. 98, No. 10, 2006, pp. 1393-1395. doi:10.1016/j.amjcard.2006.06.033

[19] J. H. Lee, J. H. O'Keefe, C. J. Lavie, R. Marchioli and W. S. Harris, "Omega-3 Fatty Acids for Cardioprotection," Mayo Clinic Proceedings, Vol. 83, No. 3, 2008, pp. 324332. doi:10.4065/83.3.324

[20] C. M. Albert, H. Campos, M. J. Stampfer, et al., "Blood Levels of Long-Chain n-3 Fatty Acids and the Risk of Sudden Death," New England Journal of Medicine, Vol. 346, No. 15, 2002, pp. 1113-1138. doi:10.1056/NEJMoa012918

[21] W. S. Harris, "The Omega-3 Index as a Risk Factor for Coronary Heart Disease," American Journal of Clinical Nutrition, Vol. 87, No. 6, 2008, pp. 1997S-2002S.

[22] T. A. Mori and R. J. Woodman, "The Independent Effects of Eicosapentaenoic Acid and Docosahexaenoic Acid on Cardiovascular Risk Factors in Humans," Current Opinion in Clinical Nutrition \& Metabolic Car, Vol. 9, 2006, pp. 95-104. doi:10.1097/01.mco.0000214566.67439.58

[23] M. C. Morris, F. Sacks and B. Rosner, "Does Fish Oil Lower Blood Pressure? A Meta-Analysis of Controlled Trials," Circulation, Vol. 88, No. 2, 1993, pp. 523-533.

[24] R. S. Dokholyan, C. M. Albert, L. J. Appel, et al., “A Trial of Omega-3 Fatty Acids for Prevention of Hypertension," American Journal of Cardiology, Vol. 93, No. 8, 2004, pp. 1041-1043. doi:10.1016/j.amjcard.2004.01.013

[25] M. L. De Rosa, et al., "Obesity, Weight Loss and Hypertension, Abstract of ASH, New York, 14-18 May 2005," American Journal of Hypertension, Vol. 18, No. 5, 2005, p. 196A.

[26] M. L. De Rosa, et al., "Long-Term Risk of Hypertension and Weight Loss in Overweight Adults," Journal of Clinical Hypertension, 2006.

[27] C. J. Liu, S. M. Conklin, S. B. Manuck, J. K. Yao and M. F. Muldoon, "Long-Chain Omega-3 Fatty Acids and Blood Pressure," American Journal of Hypertension, Vol. 24, No. 10, 2011, pp. 1121-1126.

[28] T. A. Mori, "Omega-3 Fatty Acids and Hypertension in Humans," Clinical and Experimental Pharmacology and Physiology, Vol. 33, No. 9, 2006, pp. 842-846. doi:10.1111/j.1440-1681.2006.04451.x 